\title{
Recent Progresses of Global Minimum Searches of Nanoclusters with a Constrained Basin-Hopping Algorithm in the TGMin Program
}

\author{
Xin Chen ${ }^{\mathrm{a}}$, Ya-Fan Zhao ${ }^{\mathrm{a}, \mathrm{b}, \mathrm{c}, *}$, Lai-Sheng Wang ${ }^{\mathrm{d}, *}$, Jun $\mathrm{Li}^{\mathrm{a}, *}$ \\ ${ }^{a}$ Department of Chemistry and Key Laboratory of Organic Optoelectronics \& Molecular Engineering \\ of Ministry of Education, Tsinghua University, Beijing 100084 (China) \\ ${ }^{\mathrm{b}}$ Institute of Applied Physics and Computational Mathematics, Beijing 10088 (China) \\ ${ }^{\mathrm{c}}$ CAEP Software Center for High Performance Numerical Simulation, Beijing 10088 (China) \\ ${ }^{\mathrm{d}}$ Department of Chemistry, Brown University, Providence, Rhode Island 02912 (USA) \\ *E-mail: zhao_yafan@iapcm.ac.cn; Lai-Sheng_Wang@brown.edu; junli@tsinghua.edu.cn
}

\begin{abstract}
Finding the global minima of nanoclusters is of great importance in cluster science and nanoscience. We have developed an efficient global minimum search program, named Tsinghua Global Minimum (TGMin), based on the Basin-Hopping algorithm to find the global minima of nanoclusters, as well as periodic systems. We have recently made several improvements to the original Basin-Hopping algorithm, including a constrained perturbation function, a covalent-radius-based relaxation algorithm, an improved ultrafast shape recognition algorithm, and a planeness-check mechanism. The TGMin program has been successfully applied to search the global minima of a number of nanoclusters and periodic structures, including $\mathrm{B}_{30}, \mathrm{~B}_{35}, \mathrm{~B}_{36}, \mathrm{~B}_{39}, \mathrm{~B}_{40}, \mathrm{CoB}_{18}{ }^{-}, \mathrm{RhB}_{18}{ }^{-}, \mathrm{MnB}_{16}{ }^{-}$, and $\mathrm{Au}_{7}$ on the $\alpha-$ $\mathrm{Al}_{2} \mathrm{O}_{3}(0001)$ surface. An overview of the TGMin code and several of its recent applications are presented here.
\end{abstract}




\section{Introduction}

Nanoclusters have attracted wide attentions because of their novel size-dependent properties and potentials for applications in materials science and catalyses. Nanoclusters can display totally different and tunable physical and chemical properties realtive to those of the bulk, simply by changing the cluster size. Determination of the global minimum structure is the key step to identify properties of nanoclusters, but this is still a major challenge because exploring the full potential energy surfaces of nanoclusters is extremely computationally expensive. A variety of global minimum search algorithms and methods have been proposed to overcome this challenge, including the statistical mechanical methods [1], the global reaction route mapping (GRRM) strategy [2-4], the genetic algorithm (GA) [5-7], the particle swarm optimization algorithm (PSO) [8,9], the stochastic surface walking (SSW) algorithm [10], the Kick method [11,12], the minima hopping approach [13], and the Basin-Hopping (BH) algorithm [14-16]. It is necessary to choose a suitable algorithm for different systems based on the physical and chemical properties and the computational cost. For large systems with structures close to their bulk materials, the statistical mechanical methods may be a better choice. On the other hand, for small clusters with potential energy surfaces that can be reconstructed with affordable and accurate $a b$ initio mehtods, the GRRM strategy may be preferred, which can also yield information about transition states. For nanoclusters with tens of atoms, however, their potential energy surfaces are too complicated to be investigated in detail. The popular Basin-Hopping algorithm, which does not need to traverse the detailed potential energy surface, may be the method of choice for such systems. Several global optimization programs based on the $\mathrm{BH}$ algorithm have been developed, in conjuction with DFT methods [17-19], DFTB methods [20,21] or (semi-)empirical [22,23] potentials for local geometry optimization.

The current work presents an enhanced $\mathrm{BH}$ algorithm based on the Python program for searching global minima of nanoclusters, as well as for periodic systems. The article is organized as the following: the enhanced algorithm will be introduced in Section 2 and a few recent applications of the algorithm will be described in Section 3. A brief conclusion is given in Section 4.

\section{Algorithm}

The Tsinghua Global Minimum (TGMin) package [24] is based on the BH algorithm, in which an initial locally-optimized seed structure, $X_{\text {seed }}$, is required. A new structure $X_{k}$ will then be generated from the seed structure: $X_{k}=X_{\text {seed }}+\lambda d_{k}$, where $d_{k}$ is a displacement vector 
and $\lambda$ is the step size. Let $X_{k}^{\prime}$ represent the locally optimized structure and it will only be accepted as a new seed structure if $E\left(X_{k}^{\prime}\right)<E\left(X_{\text {seed }}\right)$ or the Metropolis acceptance criterion

$$
p=\exp \left\{-\left[E\left(X_{k}^{\prime}\right)-E\left(X_{\text {seed }}\right)\right] / \mathrm{k}_{\mathrm{b}} T\right\}>p_{k}
$$

is satisfied, where $p_{k}$ is a number randomly selected from the uniform distribution of $[0,1]$, $\mathrm{k}_{\mathrm{B}}$ is the Boltzmann constant and $T$ is the effective temperature used to control the acceptance ratio. Otherwise, $X_{\text {seed }}$ remains the seed structure for several more iterations. With higher $T$, high energy structures will have more probability to be accepted as the new seed structures.

The $\mathrm{BH}$ algorithm is highly efficient and easy to implement for relatively small clusters. However, for large clusters it has several drawbacks. First, the step size $\lambda$ has to be properly set. If $\lambda$ is too small, the new structure $X_{k}^{\prime}$ optimized from the perturbed structure $X_{k}$ may be the same as the seed. If the $\lambda$ is too large, $X_{k}$ may change drastically, leading to unreasonable high-energy structures, which may require much more computing time in geometry optimization. Second, duplicated structures may occur during the global optimization. If the displacement step is too small, the perturbed structure $X_{k}$ may go back to its seed structure $X_{\text {seed }}$ after geometry optimization. The perturbed structures $X_{i}$ and $X_{j}$ may also be the same if no similarity check (Section 2.3) is performed. The third problem is that the perturbed structures $X_{k}$ should be physically reasonable: the atoms in these clusters should not be too close or too far from each other. The last problem is that the $\mathrm{BH}$ algorithm is a relatively local algorithm. For complicated potential energy surfaces with several funnels, if one starts from a structure in one funnel, it may be difficult to find more stable structures in another funnel. To overcome these problems, we have added several constraints to the original $\mathrm{BH}$ algorithm in our TGMin code, as described below.

\subsection{Constrained perturbation algorithm}

The best perturbation algorithm should generate new structures, which are sufficiently different and also close to the seed structure so that the BH search can jump out of the current basin, but do not hop too far from the seed. In our program, the displacements of the atoms are controlled by the maximum step size $\lambda$ and displacement angle $\theta$. The definition of $\theta$ is given by Fig. 1. The angle is required to be within a given range. If it is too small, atoms will move outwards; If it is too large, atoms will move inwards. Typically $\theta$ (in degrees) is randomly drawn from the uniform distribution of $[60.0,100.0]$. In the case of global minimum search for cage-like clusters ( $B_{40}$ for instance), the allowed range of $\theta$ is usually between 75 and 105 degrees, so the displacement vectors do not break the cage structure. 
According to our experience, nanoclusters with more low-coordinatition atoms tend to have higher energies. Hence, we have applied a new constraint according to this assumption: only atoms with low coordination number are allowed to be perturbed.

\subsection{Covalent-radii based relaxation}

Not all structures generated from the perturbation algorithm are chemically reasonable. Some atoms may be too close to other atoms, while some may be too far from the others. These unreasonable structures can cause severe SCF convergence problems during geometry optimization using some DFT programs. A reasonable distance between two atoms should be close to the sum of their covalent radii [25-27]. Hence, we have implemented a pre-relaxation algorithm to make sure each atom has a reasonable distance with at least one atom. For two atoms $i$ and $j$, let $r_{i}$ and $r_{j}$ be their covalent radius, $d_{i j}$ be their distance and $d_{t o l}$ be a small distance tolerance. If $d_{i j}<r_{i}+r_{j}+d_{t o l}$, atoms $i$ and $j$ are considered to be adjacent. If $d_{i j}<r_{i}$ $+r_{j}-d_{t o l}$, atom $i$ and $j$ are considered to be too close and a virtual spring force will be applied to push atoms $i$ and $j$ away from each other. After the pre-relaxation, all atomic distances should be reasonable and the SCF convergence can be greatly improved.

\subsection{Similarity check algorithm}

To avoid duplicated runs during the global minimum search, we have implemented a modified version of the Ultrafast Shape Recognition algorithm (USR) [28-30] to compare the similarities of the structures. The original USR algorithm uses 12 descriptors as the fingerprints for each structure. The similarity of two structures $\mathbf{1}$ and $\mathbf{2}$ are obtained by the equation:

$$
S^{1,2}=\frac{1}{1+\frac{1}{12}\left(\sum_{i=1}^{12}\left|d_{i}^{1}-d_{i}^{2}\right|\right)}
$$

where $d^{1}$ and $d^{2}$ are the USR descriptors for structures $\mathbf{1}$ and 2.

The standard USR algorithm uses only coordinates, which works well for clusters with only one type of atomic species. But for bimetallic or more complicated clusters, the USR algorithm may fail to identify different structures. For example, the two $\mathrm{Au}_{9} \mathrm{Zn}$ clusters in Fig. 2 are not distinguishable by the standard USR algorithm.

We have added another 12 descriptors calculated from the coordinates of a selected atomic species. The equation then becomes: 


$$
S^{1,2}=\frac{1}{1+\frac{1}{24}\left(\sum_{i=1}^{12}\left|d_{i}^{1}-d_{i}^{2}\right|+\sum_{i=1}^{12}\left|d_{i}^{M, 1}-d_{i}^{M, 2}\right|\right)}
$$

where $d^{M, 1}$ and $d^{M, 2}$ are the extra USR descriptors of structures 1 and 2 using the coordinates of atom $\mathrm{M}$.

With this improvement, we can get a better accuracy for filtering out duplicated isomers.

\subsection{Planeness-check algorithm}

In the standard $\mathrm{BH}$ algorithm, only the energies of the structures are included in the Metropolis acceptance criterion. Other chemical properties, such as the structure morphology, are excluded. Some clusters, such as small gold and boron clusters, tend to have planar isomers rather than three-dimensional structures. In the case of the $\mathrm{B}_{20}$ cluster [31], the most stable isomer is a $D_{10 d}$ drum structure while other low-lying isomers are all planar. Thus, global minimum searches of the planar and non-planar structures should be run separately. For a cluster with its three principle rotation inertia $\mathbf{I}_{\mathrm{a}}, \mathbf{I}_{\mathrm{b}}$ and $\mathbf{I}_{\mathrm{c}}\left(\mathbf{I}_{\mathrm{a}} \leq \mathbf{I}_{\mathrm{b}} \leq \mathbf{I}_{\mathrm{c}}\right)$, the planeness $P$ can be expressed by:

$$
P=\frac{I_{c}}{I_{a}+I_{b}}
$$

If the structure is completely planar, $P=1.0$. Otherwise $P$ is smaller than 1.0. Fig. 3 shows the planeness of different isomers of the $\mathrm{B}_{20}$ cluster. With this parameter we can quantitatively distinguish planar and non-planar structures.

With these four constrain algorithms added, we have implemented a new BH-based global optimization Python program, called TGMin (Tsinghua Global Minimum), for effiecient global minimum searcher for nanoclusters and peridoic systems. We have also developed interfaces to ADF [32], Gaussian09 [33], and CP2K [34] for local geometry optimization and Torque/PBS, LFS for job management. The overall workflow of the constrained $\mathrm{BH}$ algorithm is illustrated in Fig. 4.

\section{Applications}

The TGMin program has been tested with numerous nanoclusters, including the wellknown $\mathrm{Au}_{20}, \mathrm{~B}_{20}$, and $\mathrm{C}_{60}$, and has been successfully applied to find the global minima of many nanoclusters and periodic structures. A few selected examples are presented here. 


\section{1. $C_{60}$}

The famous $\mathrm{C}_{60}$ fullerene cluster has a soccer-ball shape with a very high $I_{h}$ symmetry [35]. We conducted a global minimum search test of $\mathrm{C}_{60}$ with TGMin using the CP2K program and DFTB method with the planeness check disabled. The initial seed was a randomly generated structure with $C_{1}$ symmetry. The maximum displacement step size was set to be $0.80 \AA$ and the displacement angle range was between 80 and 100 degrees. We found the $I_{h}$ hsymmetry fullerene structure after about 10,000 structures were optimized. The lowest energy of each Monte Carlo step is shown in Fig. 5.

\section{2. $A u_{20}$}

The $T_{d}$ symmetry tetrahedral $\mathrm{Au}_{20}$ cluster was first reported by Li et al. in 2003 with limited structural optimization [36], in conjunction with a very characteristic photoelectron spectrum of $\mathrm{Au}_{20}{ }^{-}$, which suggested a highly stable and possibly symmetric neutral $\mathrm{Au}_{20}$. We did a test global minimum search for $\mathrm{Au}_{20}$ starting from a randomly constructed 3D structure. The geometry optimization was done by the CP2K package at the PBE level [37] with the GTH potential [38] and the MOLOPT DZVP basis set [39]. The energy cutoff used was 280 Ry. The maximum displacement step size for $\mathrm{Au}_{20}$ was $2.7 \AA$. The minimum coordination number for perturbing an atom was set to be 7. The deep-lying tetrahedral global minimum was found in only 36 Monte Carlo steps, as shown in Fig. 6.

\section{3. $B_{20}$}

The $B_{20}$ cluster was considered to be the possible transition point between $2 \mathrm{D}$ and $3 \mathrm{D}$ structures for neutral boron clusters $[31,40]$. We searched the global minimum of $B_{20}$ with the same DFT method used for $\mathrm{Au}_{20}$. Starting from a random three-dimensional structure with the planeness check disabled, we found the $D_{10 d}$ drum-like (tubular) structure of $\mathrm{B}_{20}$ (Fig. 7) after about 870 Monte Carlo steps. As a comparison, with the maximum planeness set to be 0.870 , we were able to find the $D_{10 d}$ structure within 424 steps from the same initial seed. The low-lying isomers of $\mathrm{B}_{20}$ are given in Fig. 7. It should be pointed out that the tubular $\mathrm{B}_{20}{ }^{-}$ anion was also found to be the global mimimum, but the exeprimentally observed $\mathrm{B}_{20}{ }^{-}$was a planar isomer [31]. This cunudrum has not been resolved and has been discussed in more detail in ref. [40].

The correlations of the relative energies of the low-lying isomers and their planeness are presented in Fig. 8. It seems that structures with higher planeness tend to have lower 
energies, but the global minimum $D_{10 d}$ structure is not a planar structure. The global minimum search can be accelerated significantly if the planeness range can be set properly.

\section{4. $B_{35}$ and $B_{36}$}

The $\mathrm{B}_{36}$ cluster was the first boron cluster whose global minimum structure was found by TGMin [41], following initial searches using the Cartesian Walking method that was primarily foucsed on planar strucutres [42]. Prelimianr searches using the Cartisian Walk method found a planar $\mathrm{B}_{36}{ }^{-}$structure with a hexagonal vacancy. Subsequently, TGMin was used for unbiased searches to locate the global minium of $\mathrm{B}_{36}{ }^{-}$and confirmed the hexagonal structure of $\mathrm{B}_{36}{ }^{-}$. Geometry optimization was done by the $\mathrm{CP} 2 \mathrm{~K}$ package at the PBE level with the GTH potential and the MOLOPT DZVP basis set. The global minimum of $\mathrm{B}_{36}$ and $\mathrm{B}_{36}{ }^{-}$were found to be both quasi-planar structures: the neutral $\mathrm{B}_{36}$ has $C_{6 v}$ symmetry and the anion is slightly distorted to $C_{2 v}$ symmetry. The neutral $\mathrm{B}_{36}$ has a perfect hexagonal symmetry and can be viewed as a motif for extended monolayer boron, a new 2D mateiral named as "borophene" by Piazza et al. (Fig. 9) [41]. The global minimum of $\mathrm{B}_{35}{ }^{-}$is very similar to $\mathrm{B}_{36}$, but has one more hexagonal vacancy [43] (Fig. 10), which was also found by the TGMin code. The planar $B_{35}$ cluster was shown to be an even more flexible motif to build borophenes with different hexagonal hole patterns an densityies [43].

\section{5. $B_{40}$}

The $\mathrm{B}_{40}$ cluster was the first all-boron fullerene-like cluster (named borospherene) discovered using the global minimum search by TGMin [44], following the investigation on $\mathrm{B}_{36}$ and $\mathrm{B}_{36}{ }^{-}$[41]. Photoelectron spectra of the $\mathrm{B}_{40}{ }^{-}$cluster revealed two co-existing isomers. TGMin searches found two close-lying strucutres competing for the global minium: a quasiplanar structure with $C_{s}$ symmetry and a fullerene-like cage with $D_{2 d}$ symmetry (Fig. 11). The calculated vertical detachment energies (VDEs) and adiabatic detachment energies (ADEs) for the low-lying structures agree with the experimental data from photoelectron spectroscopy (PES). The $\mathrm{B}_{40}$ borospherene has 16 tetracoordinated and 24 pentacoordinated boron atoms, and consists of two hexagonal and four heptagonal holes on its surface [44]. More than 5000 unique structures, including the fullerene-like cage and the quasi-planar structure, were obtained during the global minimum searches (planar search and 3D search) by TGMin using CP2K at the PBE level with GTH potential and MOLOPT DZVP basis set. The TGMin resutls were also confirmed by global searches usign SSW [44]. 


\section{6. $B_{30}{ }^{-}$and $B_{39}{ }^{-}$}

TGMin has been used to locate the global minima of several boron clusters following its successes in finding the borophene-type $\mathrm{B}_{36}$ and $\mathrm{B}_{35}$ clusters and the first borospherene. Here, we highlight two more interesitng examples. Using TGMin, we found the first chiral boron clusters: the quasi-planar $\mathrm{B}_{30}{ }^{-}$[45] and the $\mathrm{B}_{39}{ }^{-}$borospherene [46]. The most stable structure of $\mathrm{B}_{30}{ }^{-}$was found to be quasi-planar with a hexagonal hole and a pair of enantiomers arising from different positions of the hexagonal hole. The global minimum of $\mathrm{B}_{39}{ }^{-}$was a chiral $C_{3}$ borospherene consisting of three hexagons and three heptagons around the $C_{3}$ axis (Fig. 12). A close-lying chiral $C_{2}$ cage isomer was also found for $\mathrm{B}_{39}{ }^{-}[46]$.

\subsection{Bimetallic boron clusters}

TGMin has also been shown to be efficient in the global minimum searches of bimetallic boron clusters, such as $\mathrm{MnB}_{16}{ }^{-}$[47], $\mathrm{RhB}_{18}{ }^{-}$[48], and $\mathrm{CoB}_{18}{ }^{-}$[49]. The global minimum changes from 3D drums for $\mathrm{CoB}_{16}{ }^{-}$[50] and $\mathrm{MnB}_{16}{ }^{-}$to a quasi-planar species for $\mathrm{CoB}_{18}{ }^{-}$. The most stable structure of $\mathrm{MnB}_{16}{ }^{-}$is a $C_{4 v}$ drum with two $\mathrm{B}_{8}$ rings, similar to $\mathrm{CoB}_{16}{ }^{-}$, while $\mathrm{RhB}_{18}{ }^{-}$has two co-existing stable isomers: one $D_{9 d}$ drum with two $\mathrm{B}_{9}$ rings and another quasi-planar structure with $C_{s}$ symmetry. For $\mathrm{CoB}_{18}{ }^{-}$, the most stable isomer becomes a quasi-plane (metallo-borophene) with $C_{2 v}$ symmetry. The photoelectron spectrum of $\mathrm{RhB}_{18}$ is compared with simulated spectra of the drum and 2D isomers in Fig. 13. Such good agreement between expeirment and theory reaffirms the global minima and low-lying isomers from the TGMin global searches. The geometry optimization for the bimetallic boron clusters were carried out by ADF at the PBE/DZ level. Obtained low-lying isomers within $1.5 \mathrm{eV}$ were usually optimized again at higher accuracy by ADF at the PBE0/TZP level and their relative energies were calculated at $\operatorname{CCSD}(\mathrm{T})$ level by Molpro [51] program. The higher level calculations are necessary to confirm the global minimum, in particular for the comparison with experiment.

\section{8. $\mathrm{Au}_{7}$ on $\square-\mathrm{Al}_{2} \mathrm{O}_{3}(0001)$ surface}

TGMin was designed not only for global minimum searches for nanoclusters, but also for periodic structures. Liu and co-workers used TGMin with several chemical constraints and successfully found the global minimum structures of the $\mathrm{Au}_{7}$ cluster on different alumina surfaces [52], as shown in Fig. 14. The optimization was carried out by CP2K at the PBE level with the GTH pseudopotential and the MOLOPT DZVP basis set. 


\section{Conclusions}

We have developed an efficient global minimum search program, TGMin, based on the Basin-Hopping algorithm with several judiciously chosen constraints: a coordination number related perturbation algorithm, a covalent radii based relaxation algorithm, an improved ultrafast shape recognition algorithm for removing structure duplications, and a parameter for planar and nonplanar searching. By adding these chemically-motivated constraints, the TGMin program has been shown to be quite efficient in predicting global minima of not only nanoclusters, but also for periodic surface structures. A number of recent examples have been used to illustrate the power of TGmin. It is expected that TGMin will signficantly accelerate the global minimum searches of larger and more complicated nanoclusters and nanostructures.

\section{Acknowledgements}

The calculations were performed using the Center for Computation and Visualization (CCV) at Brown University, the Supercomputer Center of the Computer Network Information Center at the Chinese Academy of Sciences and the Molecular Science Computing capability at the EMSL, a national scientific user facility sponsored by the Department of Energy's Office of Biological and Environmental Research and located at PNNL, a multiprogram national laboratory operated for the Department of Energy by Battelle and the Computational Lab of Department of Chemistry at Tsinghua University. Y.F.Z acknowledges funding support from National Key Research and Development Program of China under Grants No. 2016YFB0201203 and National High Technology Research and Development Program of China under Grant No. 2015AA01A304. L.S.W. wishes to thank the U.S. National Science Foundation for support on the studies of boron clusters and nanostructures (CHE-1632813).

\section{References}

[1] M. Polak, L. Rubinovich, Prediction of compositional ordering and separation in alloy nanoclusters, Surf. Sci. 584 (2005) 41-48.

[2] K. Ohno, S. Maeda, A scaled hypersphere search method for the topography of reaction pathways on the potential energy surface, Chem. Phys. Lett. 384 (2004) 277-282.

[3] S. Maeda, K. Ohno, A new global reaction route map on the potential energy surface of $\mathrm{H}_{2} \mathrm{CO}$ with unrestricted level, Chem. Phys. Lett. 460 (2008) 55-58. 
[4] S. Maeda, K. Ohno, K. Morokuma, Systematic exploration of the mechanism of chemical reactions: the global reaction route mapping (GRRM) strategy using the ADDF and AFIR methods, Phys. Chem. Chem. Phys. 15 (2013) 3683-3701.

[5] D.M. Deaven, K.M. Ho, Molecular geometry optimization with a genetic algorithm, Phys. Rev. Lett. 75 (1995) 288-291.

[6] D.M. Daven, N. Tit, J.R. Morris, K.M. Ho, Structural optimization of Lennard-Jones clusters by a genetic algorithm, Chem. Phys. Lett. 256 (1996) 195-200.

[7] J.B.A. Davis, A. Shayeghi, S.L. Horswell, R.L. Johnston, The Birmingham parallel genetic algorithm and its application to the direct DFT global optimisation of $\operatorname{Ir}_{N}(N=10-20)$ clusters, Nanoscale 7 (2015) 14032-14038.

[8] S.T. Call, D.Y. Zubarev, A.I. Boldyrev, Global minimum structure searches via particle swarm optimization, J. Comput. Chem. 28 (2007) 1177.

[9] Y. Wang, J. Lv, L. Zhu, Y. Ma, CALYPSO: A method for crystal structure prediction, Comput. Phys. Commun. 183 (2012) 2063-2070.

[10] C. Shang, Z.-P. Liu, Stochastic Surface Walking Method for Structure Prediction and Pathway Searching, J. Chem. Theory Comput. 9 (2013) 1838.

[11] M.A. Addicoat, G.F. Metha, Kick: Constraining a stochastic search procedure with molecular fragments, J. Comput. Chem. 30 (2009) 57-64.

[12] H. Zhai, M.-A. Ha, A.N. Alexandrova, AFFCK: Adaptive force-field-assisted ab initio coalescence kick method for global minimum search, J. Chem. Theory Comput. 11 (2015) 23852393.

[13] S. Goedecker, Minima hopping: An efficient search method for the global minimum of the potential energy surface of complex molecular systems, J. Chem. Phys. 120 (2004) 9911-9917.

[14] D.J. Wales, J.P.K. Doye, Global optimization by basin-hopping and the lowest energy structures of Lennard-Jones clusters containing up to 110 atoms, J. Phys. Chem. A 101 (1997) 5111-5116.

[15] R.P. White, H.R. Mayne, An investigation of two approaches to basin hopping minimization for atomic and molecular clusters, Chem. Phys. Lett. 289 (1998) 463-468.

[16] J.P.K. Doye, Global Optimization, Springer 2006 (New York).

[17] D.-E. Jiang, W. Luo, M.L. Tiago, S. Dai, In search of a structural model for a thiolate-protected $\mathrm{Au}_{38}$ cluster, J. Phys. Chem. C 112 (2008) 13905-13910.

[18] D.-E. Jiang, M. Walter, $A_{40}$ : A large tetrahedral magic cluster, Phys. Rev. B 84 (2011) 193402.

[19] W. Huang, A.P. Sergeeva, H.J. Zhai, B.B. Averkiev, L.S. Wang, A.I. Boldyrev, A concentric planar doubly $\pi$-aromatic $\mathrm{B}_{19}{ }^{-}$cluster, Nat. Chem. 2 (2010) 202-206.

[20] T.H. Choi, R. Liang, C.M. Maupin, G.A. Voth, Application of the SCC-DFTB method to hydroxide water clusters and aqueous hydroxide solutions, J. Phys. Chem. B 117 (2013) 51655179.

[21] T.H. Choi, Simulation of the $\left(\mathrm{H}_{2} \mathrm{O}\right)_{8}$ cluster with the SCC-DFTB electronic structure method, Chem. Phys. Lett. 543 (2012) 45-49.

[22] L.O. Paz-Borbon, T.V. Mortimer-Jones, R.L. Johnston, A. Posada-Amarillas, G. Barcaro, A. Fortunelli, Structures and energetics of 98 atom Pd-Pt nanoalloys: potential stability of the Leary tetrahedron for bimetallic nanoparticles, Phys. Chem. Chem. Phys. 9 (2007) 5202-5208. 
[23] L. Zhan, J.Z.Y. Chen, W.-K. Liu, S.K. Lai, Asynchronous multicanonical basin hopping method and its application to cobalt nanoclusters, J. Chem. Phys. 122 (2005) 244707.

[24] Y.F. Zhao, X. Chen, J. Li, TGMin: a global minimum search code based on basin hopping and divide-and-conquer strategy, Nano Res., in press.

[25] P. Pyykkö, S. Riedel, M. Patzschke, Triple-bond covalent radii, Chem. Eur. J. 11 (2005) 35113520.

[26] P. Pyykkö, M. Atsumi, Molecular single-bond covalent radii for elements 1-118, Chem. Eur. J. 15 (2009) 186-197.

[27] P. Pyykkö, M. Atsumi, Molecular double-bond covalent radii for elements Li-E112, Chem. Eur. J. 15 (2009) 12770-12779.

[28] P.J. Ballester, W.G. Richards, Ultrafast shape recognition for similarity search in molecular databases, Proc. Royal Soc. A: Math. Phy. Engi. Sci. 463 (2007) 1307-1321.

[29] P.J. Ballester, W.G. Richards, Ultrafast shape recognition to search compound databases for similar molecular shapes, J. Comput. Chem. 28 (2007) 1711.

[30] P.J. Ballester, P.W. Finn, W.G. Richards, Ultrafast shape recognition: Evaluating a new ligandbased virtual screening technology, J. Mol. Graph. Model. 27 (2009) 836-845.

[31] B. Kiran, S. Bulusu, H.J. Zhai, S. Yoo, X.C. Zeng, L.S. Wang, Planar-to-tubular structural transition in boron clusters: $\mathrm{B}_{20}$ as the embryo of single-walled boron nanotubes, Proc. Natl. Acad. Sci. (USA) 102 (2005) 961-964.

[32] G. te Velde, F.M. Bickelhaupt, E.J. Baerends, C. Fonseca Guerra, S.J.A. van Gisbergen, J.G. Snijders, T. Ziegler, Chemistry with ADF, J. Comput. Chem. 22 (2001) 931-967.

[33] M.J. Frisch, G.W. Trucks, H.B. Schlegel, G.E. Scuseria, M.A. Robb, J.R. Cheeseman, G. Scalmani, V. Barone, B. Mennucci, G.A. Petersson, H. Nakatsuji, M. Caricato, X. Li, H.P. Hratchian, A.F. Izmaylov, J. Bloino, G. Zheng, J.L. Sonnenberg, M. Hada, M. Ehara, K. Toyota, R. Fukuda, J. Hasegawa, M. Ishida, T. Nakajima, Y. Honda, O. Kitao, H. Nakai, T. Vreven, J.A. Montgomery Jr., J.E. Peralta, F. Ogliaro, M.J. Bearpark, J. Heyd, E.N. Brothers, K.N. Kudin, V.N. Staroverov, R. Kobayashi, J. Normand, K. Raghavachari, A.P. Rendell, J.C. Burant, S.S. Iyengar, J. Tomasi, M. Cossi, N. Rega, N.J. Millam, M. Klene, J.E. Knox, J.B. Cross, V. Bakken, C. Adamo, J. Jaramillo, R. Gomperts, R.E. Stratmann, O. Yazyev, A.J. Austin, R. Cammi, C. Pomelli, J.W. Ochterski, R.L. Martin, K. Morokuma, V.G. Zakrzewski, G.A. Voth, P. Salvador, J.J. Dannenberg, S. Dapprich, A.D. Daniels, Ö. Farkas, J.B. Foresman, J.V. Ortiz, J. Cioslowski, D.J. Fox, Gaussian 09, 2009.

[34] J. VandeVondele, M. Krack, F. Mohamed, M. Parrinello, T. Chassaing, J. Hutter, Quickstep: Fast and accurate density functional calculations using a mixed Gaussian and plane waves approach, Comput. Phys. Commun. 167 (2005) 103-128.

[35] H.W. Kroto, J.R. Heath, S.C. O'Brien, R.F. Curl, R.E. Smalley, C 60 : Buckminsterfullerene, Nature 318 (1985) 162-163.

[36] J. Li, X. Li, H.J. Zhai, L.S. Wang, Au 20 : A tetrahedral cluster, Science 299 (2003) 864-867.

[37] J.P. Perdew, K. Burke, M. Ernzerhof, Generalized gradient approximation made simple, Phys. Rev. Lett. 77 (1996) 3865-3868. 
[38] S. Goedecker, M. Teter, J. Hutter, Separable dual-space Gaussian pseudopotentials, Phys. Rev. B 54 (1996) 1703-1710.

[39] J. Vandevondele, J. Hutter, Gaussian basis sets for accurate calculations on molecular systems in gas and condensed phases, J. Chem. Phys. 127 (2007) 114105.

[40] L.S. Wang, Photoelectron spectroscopy of size-selected boron clusters: from planar structures to borophenes and borospherenes, Int. Rev. Phys. Chem. 35 (2016) 69-142.

[41] Z.A. Piazza, H.S. Hu, W.L. Li, Y.F. Zhao, J. Li, L.S. Wang, Planar hexagonal $B_{36}$ as a potential basis for extended single-atom layer boron sheets, Nat. Commun. 5 (2014) 3113.

[42] Z.A. Piazza, W.L. Li, C. Romanescu, A.P. Sergeeva, L.S. Wang, A.I. Boldyrev, A photoelectron spectroscopy and $a b$ initio study of $\mathrm{B}_{21}{ }^{-}$: Negatively charged boron clusters continue to be planar at 21, J. Chem. Phys. 136 (2012) 104310.

[43] W.L. Li, Q. Chen, W.J. Tian, H. Bai, Y.F. Zhao, H.S. Hu, J. Li, H.J. Zhai, S.D. Li, L.S. Wang, The $\mathrm{B}_{35}$ cluster with a double-hexagonal vacancy: A new and more flexible structural motif for borophene, J. Am. Chem. Soc. 136 (2014) 12257-12260.

[44] H.J. Zhai, Y.F. Zhao, W.L. Li, Q. Chen, H. Bai, H.S. Hu, Z.A. Piazza, W.J. Tian, H.G. Lu, Y.B. Wu, Y.W. Mu, G.F. Wei, Z.P. Liu, J. Li, S.D. Li, L.S. Wang, Observation of an all-boron fullerene, Nat. Chem. 6 (2014) 727-731.

[45] W.L. Li, Y.F. Zhao, H.S. Hu, J. Li, L.S. Wang, [B 30 ]: A quasiplanar chiral boron cluster, Angew. Chem. Int. Ed. 53 (2014) 5540-5545.

[46] Q. Chen, W.L. Li, Y.F. Zhao, S.Y. Zhang, H.S. Hu, H. Bai, H.R. Li, W.J. Tian, H.G. Lu, H.J. Zhai, S.D. Li, J. Li, L.S. Wang, Experimental and theoretical evidence of an axially chiral borospherene, ACS Nano 9 (2015) 754-760.

[47] T. Jian, W.L. Li, I.A. Popov, G.V. Lopez, X. Chen, A.I. Boldyrev, J. Li, L.S. Wang, Manganesecentered tubular boron cluster $-\mathrm{MnB}_{16}{ }^{-}$: A new class of transition-metal molecules, J. Chem. Phys. 144 (2016) 154310.

[48] T. Jian, W.L. Li, X. Chen, T.T. Chen, G.V. Lopez, J. Li, L.S. Wang, Competition between drum and quasi-planar structures in $\mathrm{RhB}_{18}{ }^{-}$: Motifs for metallo-boronanotubes and metallo-borophenes, Chem. Sci. 7 (2016) 7020-7027.

[49] W.L. Li, T. Jian, X. Chen, T.T. Chen, G.V. Lopez, J. Li, L.S. Wang, The planar $\mathrm{CoB}_{18}{ }^{-}$cluster as a motif for metallo-borophenes, Angew. Chem. Int.. Ed. 55 (2016) 7358-7363.

[50] I.A. Popov, T. Jian, G.V. Lopez, A.I. Boldyrev, L.S. Wang, Cobalt-centered boron molecular drums with the highest coordination number in the $\mathrm{CoB}_{16}{ }^{-}$cluster, Nat. Commun. 6 (2015) 8654.

[51] H.J. Werner, P.J. Knowles, G. Knizia, F.R. Manby, M. Schütz, Molpro: a general purpose quantum chemistry program package, WIREs Comput Mol. Sci. 2 (2012) 242-253.

[52] J.C. Liu, Y. Tang, C.R. Chang, Y.G. Wang, J. Li, Mechanistic insights into propene epoxidation with $\mathrm{O}_{2}-\mathrm{H}_{2} \mathrm{O}$ mixture on $\mathrm{Au}_{7} / \alpha-\mathrm{Al}_{2} \mathrm{O}_{3}$ : A hydroproxyl pathway from ab Initio molecular dynamics simulations, ACS Catal. 6 (2016) 2525-2535. 


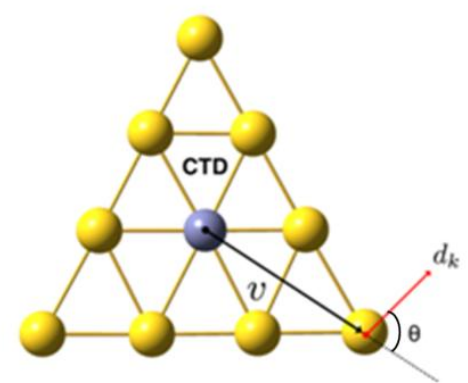

Fig. 1. The definition of displacement angle. CTD is the centroid of the cluster. $\square$ is the vector from CTD to an atom and $\square_{\square}$ is the displacement vector for this atom.

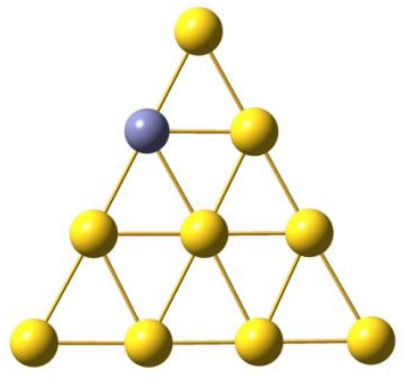

(a) $\mathrm{Zn}$ atom on the edge

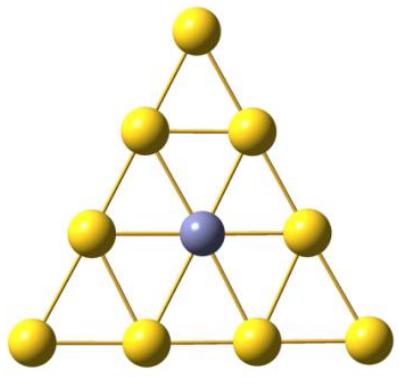

(b) $\mathrm{Zn}$ atom in the center

Fig. 2. Two isomers of the $\mathrm{Au}_{9} \mathrm{Zn}$ cluster. The golden atoms are $\mathrm{Au}$ and the blue atom is $\mathrm{Zn}$. Isomers (a) and (b) are different, but they cannot be distinguished by the standard ultrafast shape recognition algorithm. 


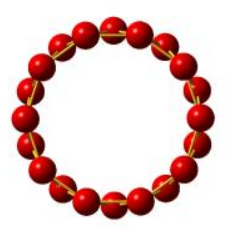

1) 0.857

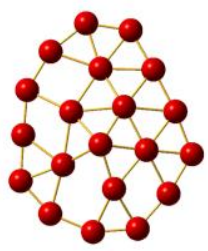

6) 0.976

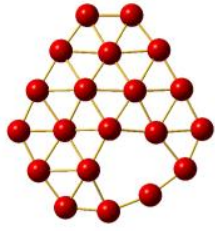

2) 0.980

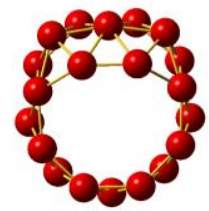

6) 0.762

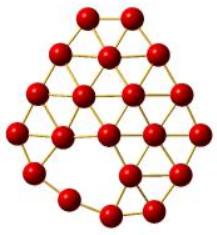

3) 0.980

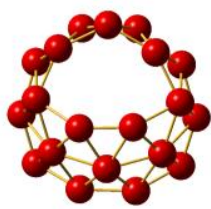

7) 0.784

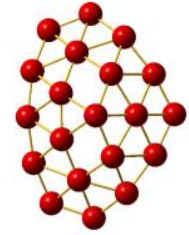

4) 0.871

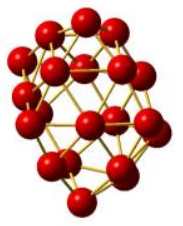

8) 0.591

Fig. 3. The planeness of different isomers of $B_{20}$.

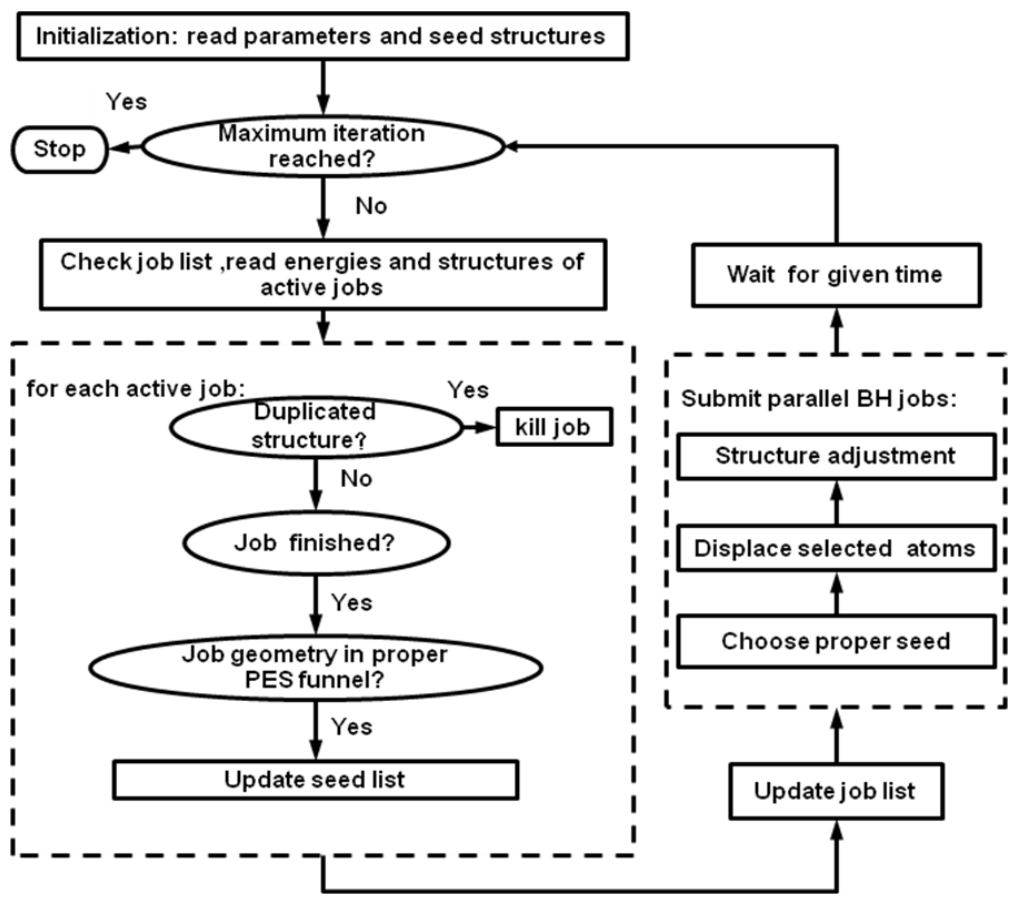

Fig. 4. The overall workflow of TGMin. 


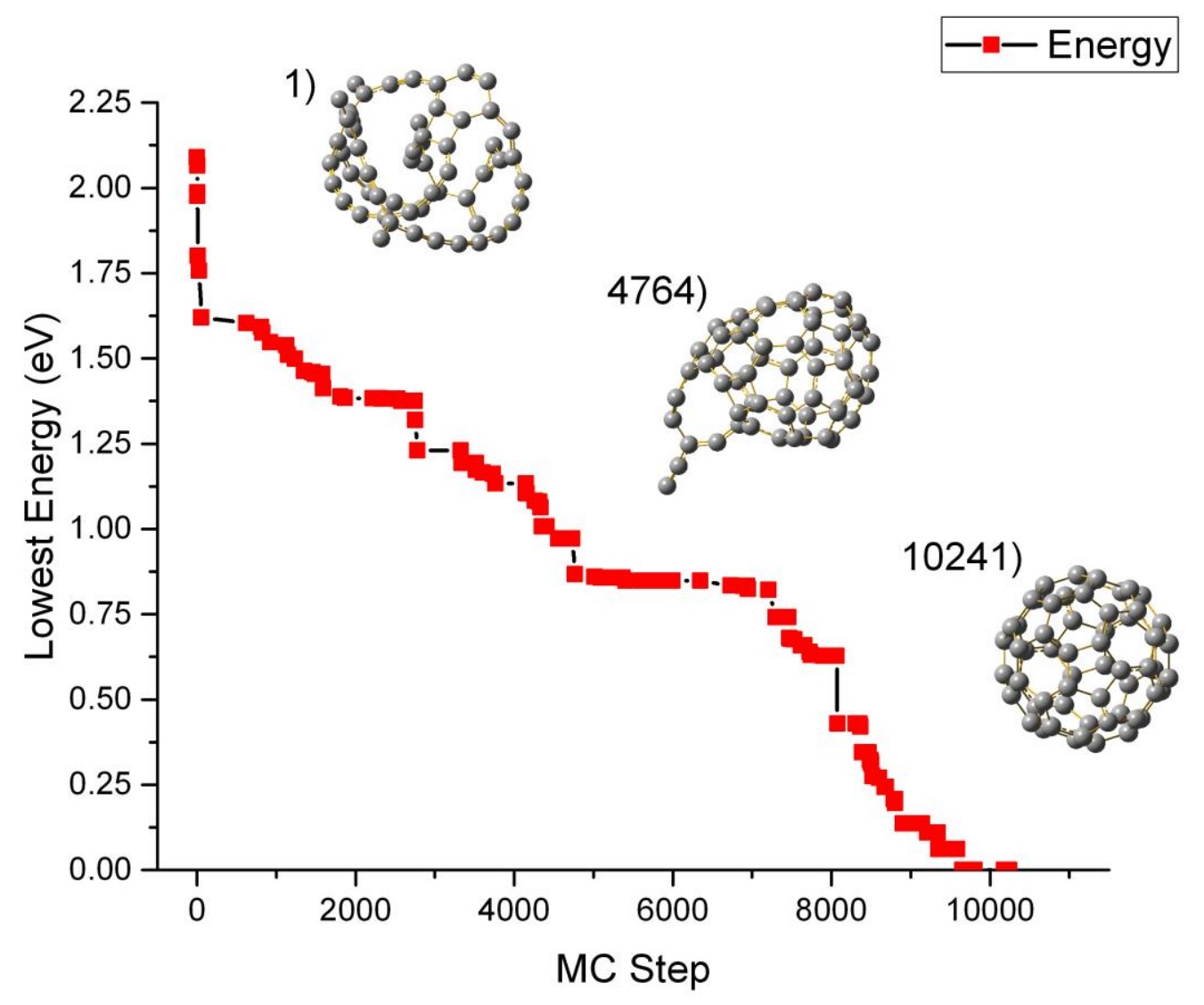

Fig. 5. The lowest energy of each Monte Carlo step during the global minimum search of $\mathrm{C}_{60}$. 


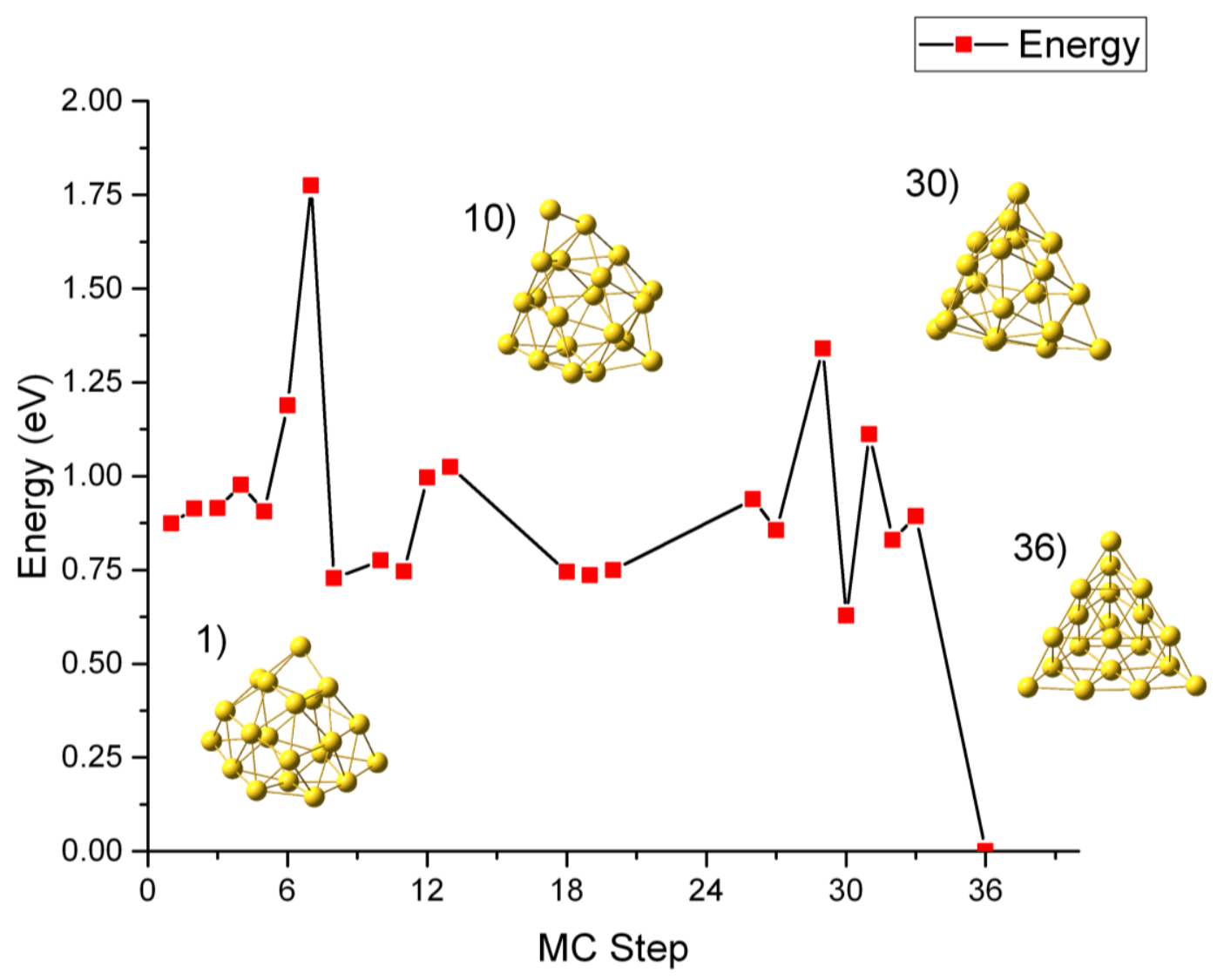

Fig. 6. The energies of the optimized structures of each Monte Carlo step during global minimum search of $\mathrm{Au}_{20}$. 


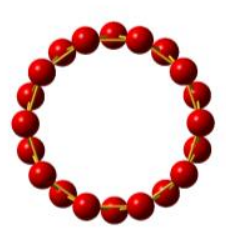

1) $0.000 \mathrm{eV}$

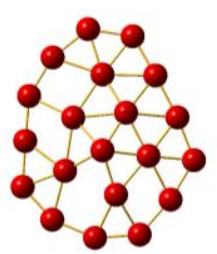

6) 0.759

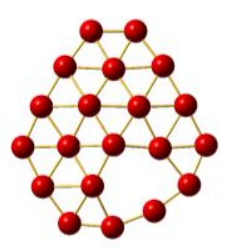

2) 0.606

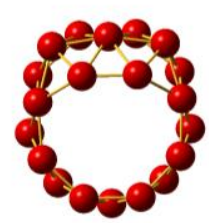

6) 0.828

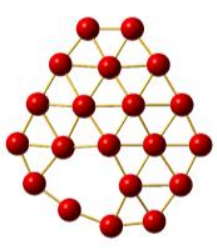

3) 0.606

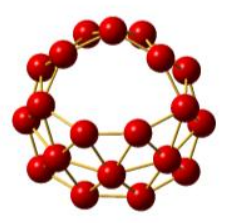

7) 0.922

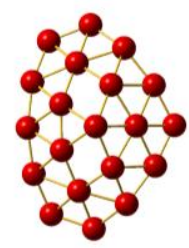

4) 0.618

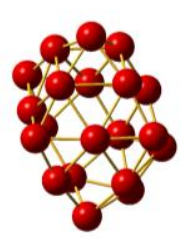

8) 1.086

Fig. 7. The low-lying isomers of $\mathrm{B}_{20}$ within $1.0 \mathrm{eV}$ of its global minimum.

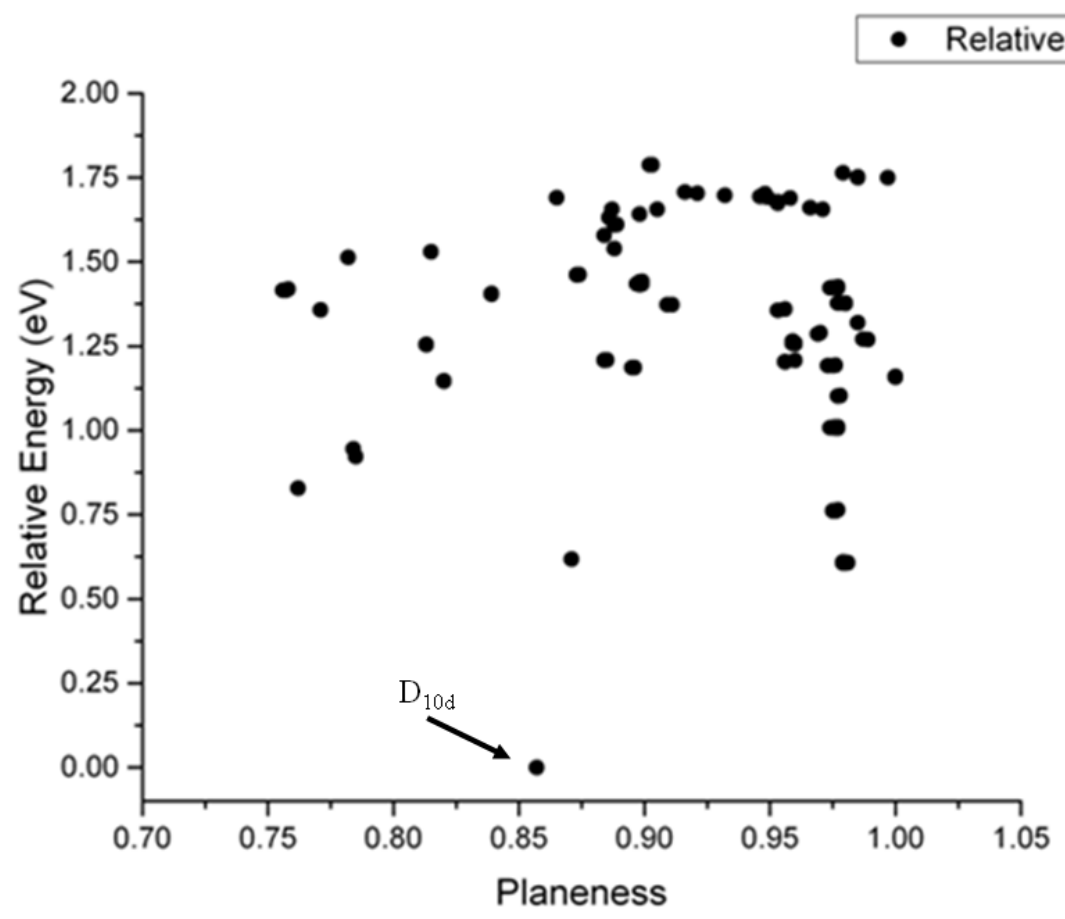

Fig. 8. Correlations of the energies within $2.0 \mathrm{eV}$ of the global minimum and the planeness of the $\mathrm{B}_{20}$ isomers. Structures with higher planeness tend to have lower energies, but the deep-lying global minimum $D_{10 d}$ structure is not a planar structure. 


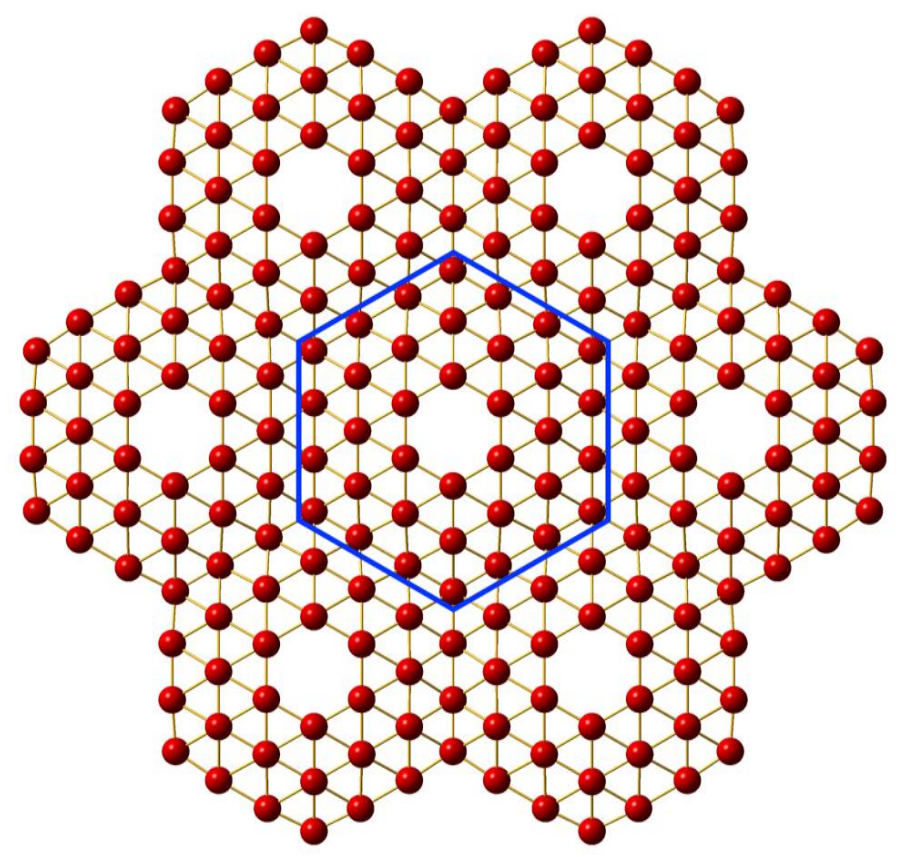

Fig. 9. The relationship between borophene and the planar hexagonal $B_{36}$ cluster. The hexagon in the blue line is a basic neutral $\mathrm{B}_{36}$ nanocluster unit.
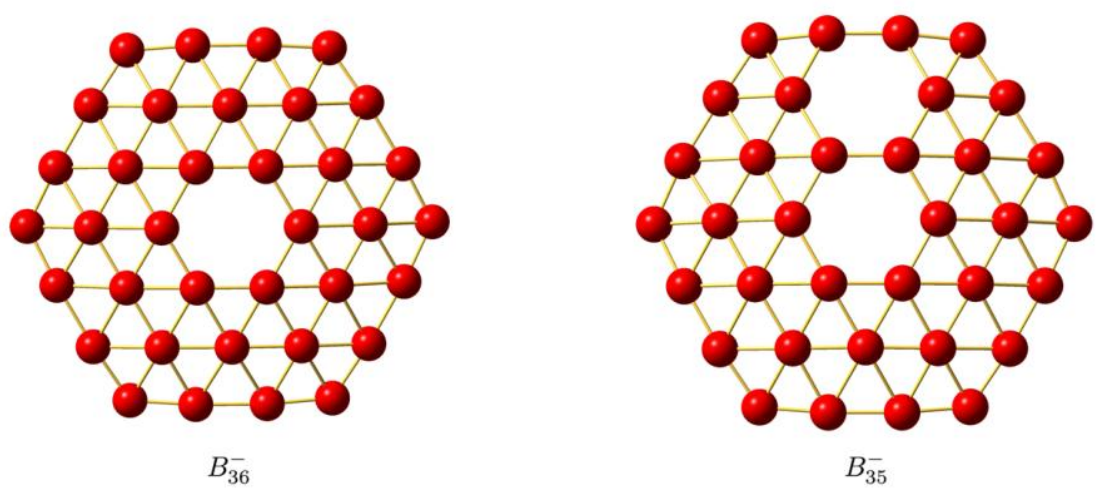

Fig. 10. Comparison of the global minima of $\mathrm{B}_{36}{ }^{-}$(left) and $\mathrm{B}_{35}{ }^{-}$(right). For both clusters, the neutral structures are similar to the anions. 


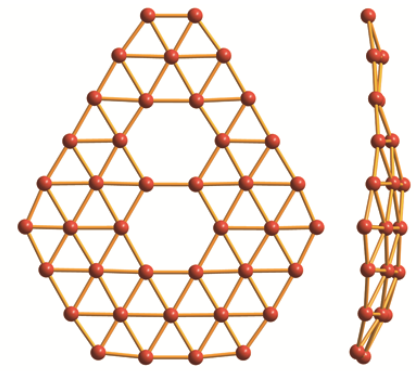

1. $C_{s} B_{40}^{-}\left({ }^{2} A^{\prime}\right)$

3. $C_{s} B_{40}\left({ }^{1} A^{\prime}\right)$
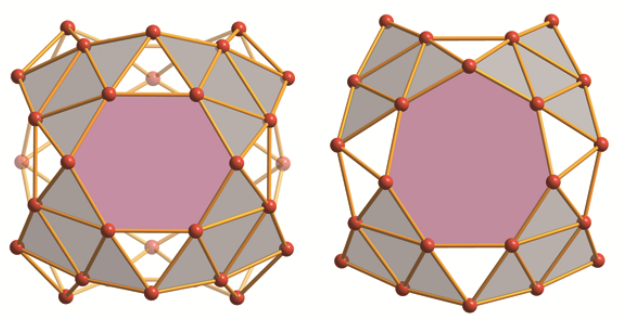

2. $D_{2 \mathrm{~d}} \mathrm{~B}_{40}{ }^{-}\left({ }^{2} \mathrm{~B}_{2}\right)$

4. $D_{2 \mathrm{~d}} \mathrm{~B}_{40}\left({ }^{1} \mathrm{~A}_{1}\right)$

Fig. 11. The top and side views of the two co-existing global minimum isomers of $\mathrm{B}_{40}{ }^{-}$. The anionic and neutral structures for both isomers are very similar with the same symmetries. The $D_{2 d} \mathrm{~B}_{40}$ cage is overwhelmingly the global minimum for the neutral and is named "borospherene".

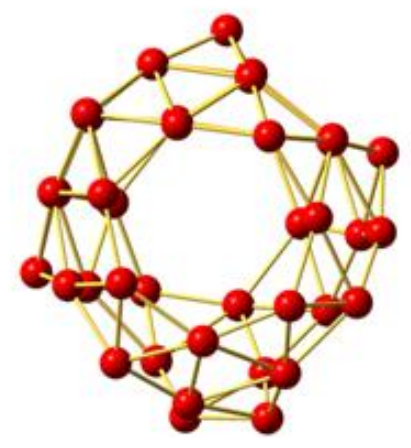

(a)

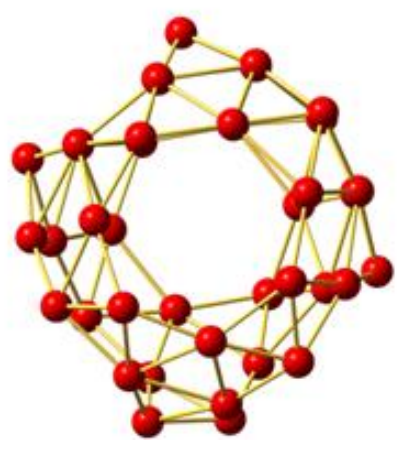

(b)

Fig. 12. The optimized global minimum structures of the $\mathrm{C}_{3}$ chiral isomers of $\mathrm{B}_{39}{ }^{-}$. Structures (a) and (b) are the two degenerate enantiomers. 


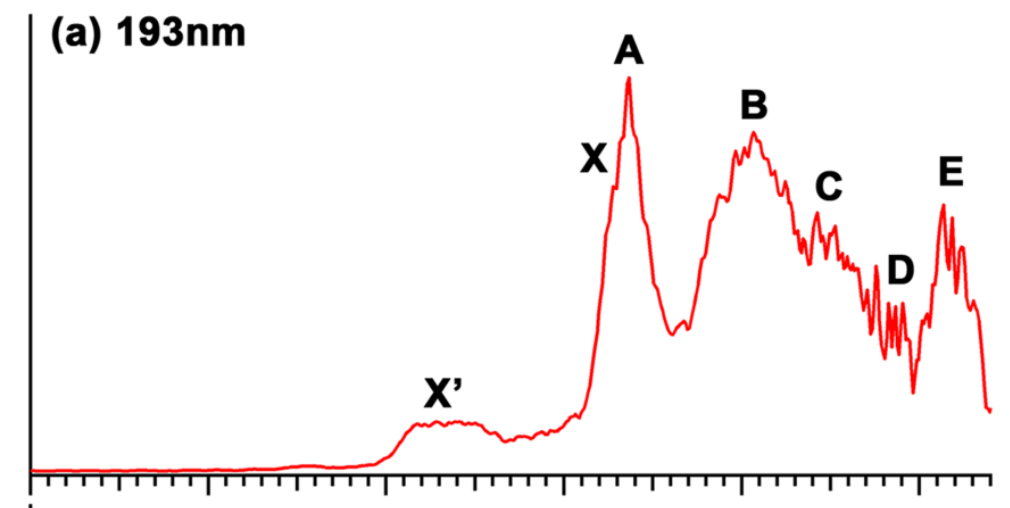

(b)

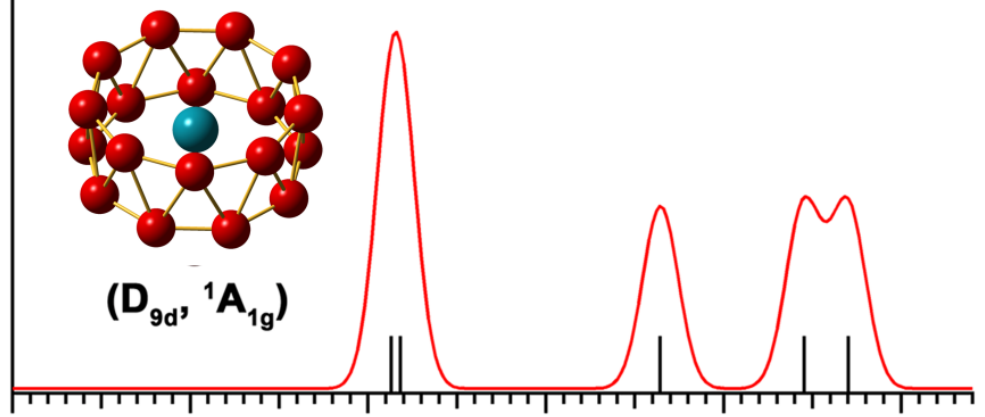

(c)

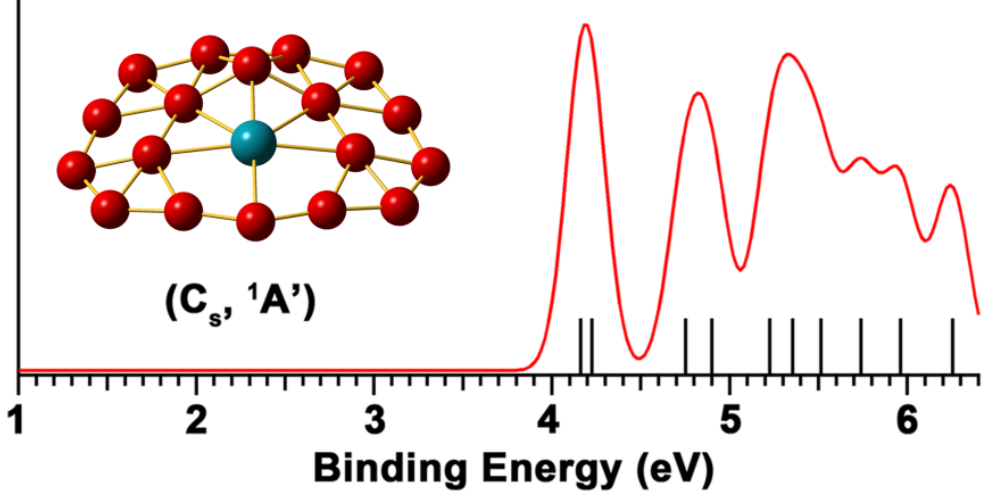

Fig. 13. The photoelectron spectrum of $\mathrm{RhB}_{18}{ }^{-}$compared with the simulated spectra of two close-lying 2D and 3D isomers. 

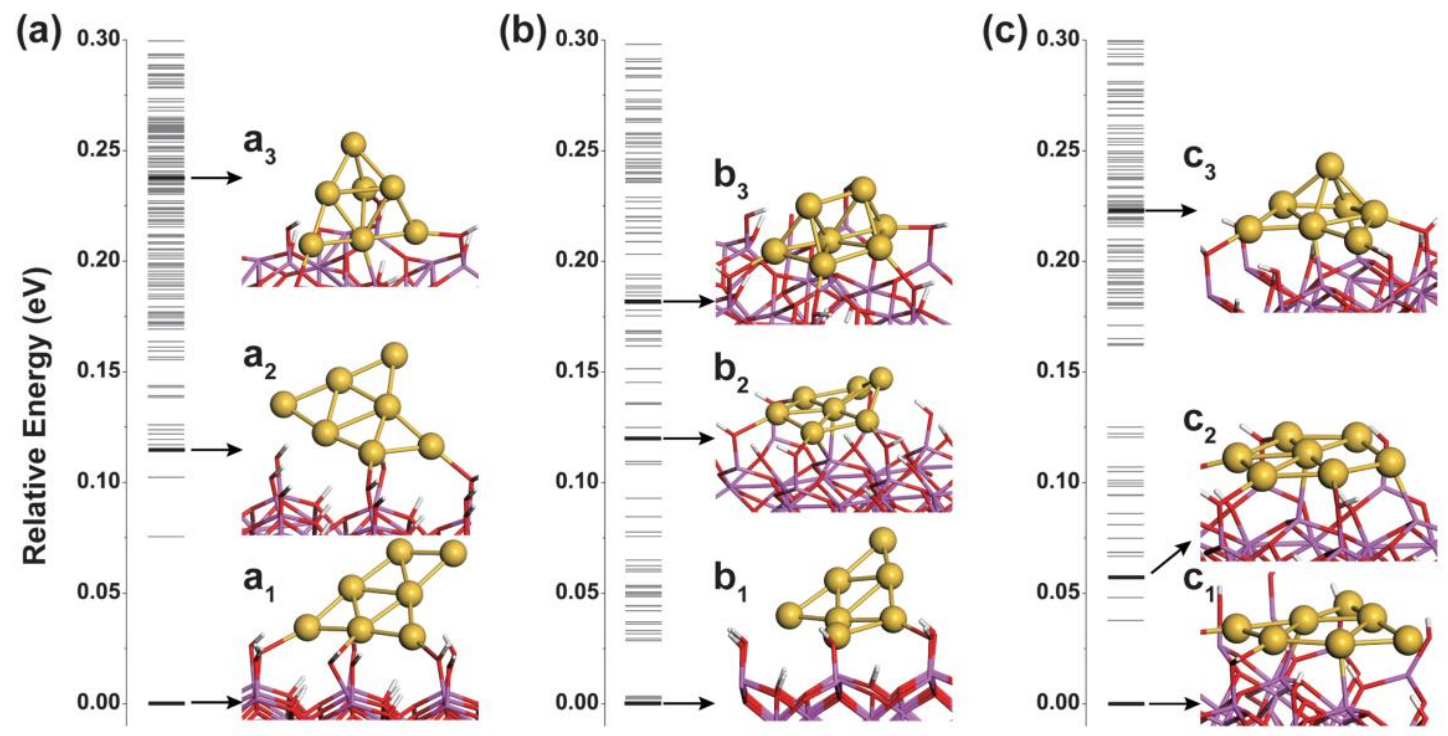

Fig. 14. Geometries and potential energies of the $\mathrm{Au}_{7}$ cluster on (a) fully hydroxylated $\alpha$ $\mathrm{Al}_{2} \mathrm{O}_{3}(0001)$ surface, (b) hydroxylated $\alpha-\mathrm{Al}_{2} \mathrm{O}_{3}(0001)$ surface missing one water molecule, and (c) hydroxylated $\alpha-\mathrm{Al}_{2} \mathrm{O}_{3}(0001)$ surface missing two water molecules. 